\title{
Potencialidad del contencioso administrativo en la eficiencia y modernización de los servicios públicos
}

\author{
Armando Rodríguez García \\ Coordinador del Postgrado en Derecho Administrativo \\ Universidad Central de Venezuela
}

Sumario: PRELIMINAR. I. LA NOCIÓN DE SERVICIO PÚBLICO COMO IDEA DE FUERZA EN LA CONSTRUCCIÓN DEL DERECHO ADMINISTRATIVO. 1. La construcción conceptual y su influencia en la configuración de la disciplina. 2. La evolución del concepto. 3. Los avances tecnológicos y su influencia. II. SERVICIO PÚBLICO, CIVILIZACIÓN, URBANIZACIÓN, TECNOLOGÍA Y CALIDAD DE VIDA.

\section{PRELIMINAR}

Pocos ámbitos de la disciplina jurídica han recibido una influencia tan decisiva de la producción jurisprudencial como sucede con el Derecho Administrativo. Indudablemente, tal circunstancia opera en atención a los espacios sustantivos de presencia que interesan a esta disciplina, impregnados por el dinamismo que identifica la realidad social en lo cotidiano, y sus transformaciones constantes.

Literalmente «construido», en sus inicios históricos, sobre la actuación sostenida y creativa del Consejo de Estado francés, a través de los múltiples arrêts; el Derecho Administrativo se nutre con instituciones nuevas, al igual que de la adaptación de diversos instrumentos producidos en el espectro propio de otras áreas del mundo jurídico.

En esta línea de actuación, las decisiones del Consejo de Estado francés son propulsoras de interpretaciones sobre normativas y técnicas jurídicas de muy diverso origen y factura, en virtud de las cuales, no sólo se ha venido cumpliendo con la función típica de control jurisdiccional sobre el ejercicio del poder y la correlativa preservación de las garantías de la libertad, el debido proceso, el derecho a la defensa y otras, en aplicación de la máxima que postula la prevalencia del Principio de Legalidad dentro de la concepción del Estado de Derecho, sino que, paralelamente, se ha moldeando un verdadero andamiaje jurídico con caracteres propios, distintivos de una parcela del conocimiento científico-jurídico, así como del actuar que corresponde a un segmento de singular importancia y dinamismo dentro del complejo enjambre de estructuras, relaciones y tareas que comporta el Estado contemporáneo, como la expresión más acabada de la sociedad civil. 
No se quiere decir con ello que la Administración pública, como realidad fenomenológica, no existiera desde etapas históricas anteriores, pues tal como señala el Profesor Massimo Severo Giannini, « todos los grupos organizados dan lugar a hechos administrativos» ${ }^{1}$; sin embargo, es indudable que la construcción dogmática y científica del Derecho Administrativo arranca a partir de la configuración del Estado Moderno, y básicamente con el establecimiento de ciertos valores fundamentales como los expresados en los Principios de legalidad, separación de Poderes y respeto a las situaciones jurídicas subjetivas.

En este sentido, la jurisdicción contencioso administrativa ha impregnado, con su actuación, el espectro general del Derecho Administrativo en todas y cada una de sus posibles manifestaciones, dentro de las cuales, como sabemos, se ubica en lugar preponderante, la noción de servicio público.

No obstante, conviene precisar que, si bien las decisiones de los tribunales contencioso administrativos tienen la potencialidad de la solución jurídica en los asuntos sometidos a su control, con fuerza definitiva, ello no significa que toda decisión jurisdiccional configure, en sí misma, un aporte singular en la percepción valorativa de una noción, en la construcción de instituciones, ni mucho menos en la indicación certera de una orientación o tendencia que marque la dirección del pensamiento científico en torno a algún tema concreto.

Por eso, hay que comenzar por advertir que, al menos por lo que corresponde al campo del Derecho Administrativo, la auténtica producción jurisdiccional, generadora de cauces sólidos de formación del pensamiento jurídico y con ello, productora de plataformas institucionales y moldes instrumentales de la estructura y funcionamiento del Estado o de los derechos de los ciudadanos, no coincide con todas las posibles sentencias que publican las diferentes instancias de decisión judicial, ni siquiera en sus niveles superiores.

Tal como sucede en términos generales en toda la extensa amplitud del campo intelectual, también en el contencioso administrativo el verdadero valor y la trascendencia de sus expresiones es exclusivamente de orden cualitativo $\mathrm{y}$, en consecuencia, descansa en la robustez, coherencia y sentido lógico de sus proposiciones, que son tales, efectivamente, en la medida en que acogen verdaderos conceptos, y no simplemente por la jerarquía formal o el efecto práctico que sobre la resolución del asunto concreto puede tener la sentencia.

Por ello, más que formular un análisis interpretativo de un elenco más o menos abultado de sentencias emitidas por los tribunales contencioso administrativos en materia de servicios públicos, estimamos procedente conside-

\footnotetext{
${ }^{1}$ Massimo Severo Giannini, Premisas sociológicas e históricas del Derecho Administrativo. Instituto Nacional de Administración Pública (INAP), Madrid, 1980, 2. ${ }^{a}$ ed.
} 
POTENCIALIDAD DEL CONTENCIOSO ADMINISTRATIVO EN ... LOS SERVICIOS PÚBLICOS

rar el peso, la potencialidad que este tipo de expresiones del quehacer jurídico puede tener en el campo sustantivo y funcional de las manifestaciones prestacionales que corresponde atender al sector público, especialmente en el ámbito local, en la medida en que el decisor judicial se abra, de manera regular y sostenida, al conocimiento del fenómeno social, económico y tecnológico que constituyen, en lo sustantivo, los servicios públicos.

De este modo será posible entender en su exacta dimensión la necesaria interdependencia de las actividades prestacionales con las manifestaciones propias de la dinámica social en la cual se insertan, inevitablemente.

Una perspectiva de este tenor conduce a una línea de valoración totalmente opuesta a la que implica refugiarse en interpretaciones legales o categorizaciones jurídicas, muchas veces anquilosadas, que obedecen a realidades conceptuales o circunstancias fácticas superadas por la evolución natural que marca la dinámica social con ritmos que incrementan exponencialmente su velocidad, y en direcciones cada vez más variadas.

Este es el patrón de aproximación que seguiremos para el tratamiento del asunto que nos ocupa. Así, partiendo de una breve revisión inicial del papel que ha representado la noción de servicio público en la construcción del Derecho Administrativo, pasamos revista a la necesaria vinculación entre las nociones de servicio público y calidad de vida, entendiendo esta última como un concepto virtual, como un umbral en que se colocan los rangos de expectativas de las comunidades para el montaje de las exigencias y la determinación de los derechos prestacionales frente al Estado.

Todo ello, a su vez, invita a contactar con conceptos tales como técnicas y modos de gestión, operatividad administrativa, calidad total, tecnología, y desde luego, urbanización, civilización, para finalmente consignar algunas reflexiones sobre la presencia del contencioso administrativo como agente o vehículo eficiente para la actualización de categorías jurídicas, prestando particular atención a la situación actual de la normativa general sobre la materia en el caso venezolano.

\section{LA NOCIÓN DE SERVICIO PÚBLICO COMO IDEA FUERZA EN LA CONSTRUCCION DEL DERECHO ADMINISTRATIVO}

\section{La construcción conceptual y su influencia en la configuración de la disciplina.}

Histórica y sustancialmente, la noción de servicio público juega un papel preponderante, y ocupa un lugar protagónico en la formación y constante actualización del Derecho Administrativo, al punto que, como todos cono- 
cemos, llega a ser en un momento dado, su noción emblemática, el concepto síntesis, en cuanto a la definición de la Administración pública, como objeto de esta rama del Derecho, considerándose ambas nociones como equivalentes o idénticas.

El espacio conceptual que llega a ocupar la noción de servicio público está directamente vinculado a su papel sustitutivo de la noción de soberanía, unido al alcance práctico que permite su contenido, montado sobre una concepción sociológica con derivaciones jurídicas, comprensiva de la actividad necesaria para el desenvolvimiento regular de las comunidades, que debe ponerse en práctica, como consecuencia de un deber correlativo a la exigencia legítima de los ciudadanos, por lo que corresponde al Estado asegurar su realización y fiscalizar las condiciones de su prestación, de lo que deriva su categorización como la «piedra angular del Derecho Administrativo», mediante la identidad de contenido conceptual a la cual hemos aludido ${ }^{2}$.

El servicio público adquiere, entonces, el valor de «noción clave», que permite integrar, bajo una aproximación sistémica, lo que hasta el momento se presentaba como una acumulación de reglas diversas, que en diferentes campos de la presencia del Estado y de la actuación de los particulares, fijaban cauces conductuales y consecuencias jurídicas las cuales debían ser seleccionadas, interpretadas y aplicadas por los ciudadanos, por los funcionarios de la Administración y por los órganos jurisdiccionales, según el caso, por lo que la operatividad práctica de su propia realidad demandaba necesariamente una referencia integradora.

Precisamente en este sentido apunta, con meridiana claridad y acierto, el Profesor Jean Rivero que: «... Se imponía, pues, un esfuerzo para desprender de esas reglas, algunos principios esenciales que permitieran organizar la materia, y de hacerla entonces, al mismo tiempo, más inteligible, más asimilable, y más utilizable.

Esta búsqueda respondía a una necesidad práctica: al lado de la jurisdicción judicial existía una jurisdicción administrativa para la cual los textos no definían con precisión las atribuciones, imponiendo al juez la definición de un criterio de competencia, de un principio general que permitiera reconocer los litigios dependientes del Derecho administrativo, e igualmente, las fronteras de la competencia de las jurisdicciones administrativas y judiciales.

En esta perspectiva, un esfuerzo doctrinal y jurisprudencial ha sido intentado, a partir de comienzo del siglo Xx, para unificar todas las reglas administrativas a partir de la noción de servicio público.

De algunas sentencias dictadas a comienzos de este siglo por el Consejo de Estado y el Tribunal de Conflictos, los principales autores de este perío-

\footnotetext{
2 Véase: Antonio Moles CAubet, «Lecciones de Derecho Administrativo». Revista de la Facultad de Ciencias Jurídicas y Políticas, No 84. Universidad Central de Venezuela, Caracas, 1992, pp.120 y stes.
} 
do - principalmente Duguit y JÉZE- han creído poder concluir que el derecho administrativo encontraba su unidad en la noción de servicio público....» ${ }^{3}$.

De momento, los datos que más nos interesa destacar a los efectos del presente ejercicio de reflexión, están centrados, por una parte, en la preponderancia de la noción de servicio público para el Derecho Administrativo, y de manera complementaria, por el papel que en su construcción y aplicación han jugado los órganos de la jurisdicción contencioso administrativa.

Esta posición de predominio conceptual, que como indicamos, llega incluso a la identificación integral e integradora de su contenido conceptual con el Derecho Administrativo, pierde terreno progresivamente, redefiniéndose su alcance y retrocediendo de la posición de vigencia absoluta de otras épocas.

No obstante, es forzoso reconocer que la noción de servicio público configura una huella de necesaria referencia en la aproximación al concepto de Administración pública, por su valor intrínseco como expresión de una modalidad de contenido de la actividad pública (si bien relativo y limitado) y por su indudable presencia histórica en la construcción del Derecho Administrativo, lo que ocurre con una muy significativa participación y aporte de las decisiones producidas por los órganos de la jurisdicción contencioso- administrativa.

\section{La evolución del concepto}

Por otra parte, es suficientemente conocida la evolución de la noción de servicio público, hacia una conceptualización más restringida y, desde luego, con menores pretensiones de omnicomprensión de lo que puede representar en su totalidad el sistema jurídico administrativo, que aquella que prevaleció en las fases iniciales de construcción de la disciplina.

Pero no es menos cierto que, en todo caso, tal evolución no ha impedido conservar el sentido de una particular forma de manifestación administrativa del Estado, asociada ineludiblemente con instrumentos, técnicas y categorías propias del Derecho Administrativo, que arrojan particularidades de singular interés para esta rama del Derecho, con un importante espacio de presencia del control contencioso administrativo, en virtud de la necesidad de resolución de los conflictos surgidos entre los múltiples y diversos actores que entrelazan sus campos de interés con motivo del diseño, instalación, operación, supervisión y control de las actividades prestacionales que se ejercitan en favor de los ciudadanos, a través de los servicios públicos, así como

\footnotetext{
3 Jean Rivero, Derecho Administrativo (Traducción de la 9a edición). Instituto de Derecho Público. Universidad Central de Venezuela. Caracas, 1984, pp. 32 y 33.
} 
para el establecimiento y aplicación general del régimen jurídico dispuesto a tal efecto.

De esta manera, a través de la noción de servicio público es factible, y en cierta medida es forzoso, entrar en contacto con diferentes categorías propias del Derecho Administrativo, dentro de las cuales se encuentra, de entrada, la noción de prestación, o actividad prestacional, en tanto forma o modalidad de actuación de la Administración Pública, regida íntegramente por el Derecho, lo que conduce directamente al abordaje y tratamiento del Principio de Legalidad y todo el formidable complejo de manifestaciones y herramientas de corte jurídico, tales como actos de autoridad (normativos y de control), actos negociales (contratos, concesiones, licitaciones, delegaciones, encomiendas etc.), situaciones jurídicas susceptibles de protección (derechos subjetivos, intereses legítimos, intereses colectivos o difusos), procedimientos administrativos, organización administrativa (ordenadora y operativa), relaciones intergubernamentales, desregulación, privatización, participación ciudadana, medios de impugnación, categorías fiscales o tributarias (tasas, precios tarifados, contribuciones especiales, impuestos), figuras de organización, controles, responsabilidad contractual y extracontractual, modernización de los métodos y sistemas administrativos, gestión y gerencia, políticas públicas, transparencia administrativa, ética pública, etc.

Adicionalmente, es claro que la marcada importancia de la noción de servicio público se amplía, se multiplica y en definitiva, se expresa, de una manera práctica, en razón a la difusión e influencia determinante que ha tenido el Derecho Administrativo francés, al inspirar el desenvolvimiento de la disciplina en otros muchos piases ${ }^{4}$.

\section{Los avances tecnológicos y su influencia}

De otra parte, las transformaciones económicas y sociales que presencia la sociedad occidental durante el último tercio del siglo XIX, especialmente en Europa y los Estados Unidos de América, como efecto de los avances tecnológicos que desembocan en la llamada Revolución Industrial, tienen, desde luego, importantes repercusiones y efectos en el campo político y jurídico, conduciendo al surgimiento de un cambio de patrón conceptual en cuanto al perfil y presencia del Estado como instrumento al servicio de la Sociedad para el mejor logro de sus fines colectivos, lo que da paso a la aparición del concepto de Estado Benefactor o Estado de Bienestar (Welfare State), en el umbral del siglo Xx.

El cambio sustancial se centra, a los efectos del tema objeto de nuestro interés inmediato, en la concepción de las tareas asumidas por el Sector Pú-

\footnotetext{
${ }^{4}$ Antonio Moles CAubet, «La progresión del Derecho Administrativo». Revista de la Facultad de Derecho. $\mathrm{N}^{\mathrm{o}}$ 1. Universidad Central de Venezuela. Caracas, 1954.
} 
POTENCIALIDAD DEL CONTENCIOSO ADMINISTRATIVO EN ... LOS SERVICIOS PÚBLICOS

blico, y particularmente por la Administración Pública, como un objetivo social, de satisfacción general de las demandas de la colectividad, en cuanto a sus condiciones de vida, en lo que concierne a la calidad de vida de los pobladores, lo que en definitiva se traduce en una transformación conceptual de las relaciones entre el Estado y la Sociedad, por la ampliación e intensificación del papel que corresponde jugar al Estado y la correlativa presencia, intensificación y amplitud en el espectro de participación para la formulación de demandas y la búsqueda de fórmulas de gestión y respuesta que asume la colectividad.

Luego, en un momento histórico más cercano, se hace presente otro importante salto tecnológico que conduce a una nueva definición de los perfiles definitorios de la sociedad y sus manifestaciones. Se trata en esta oportunidad de la revolución informática o cibernética y su impacto en las comunicaciones y el manejo de la información que se comienza a manifestar en proporciones cada vez más amplias, a partir del último tercio del siglo Xx, de modo tal que su difusión es global, mundial, ecuménica, en términos absolutamente literales.

Como consecuencia de las transformaciones anotadas, la vida social, el espacio de lo colectivo lo plural o lo público, se amplía decisivamente, frente a la reducción progresiva de lo singular, de la individualización de las soluciones o la satisfacción singular de las necesidades de los pobladores.

De este modo, la nueva Sociedad Industrial provoca y comprende el surgimiento de una nueva Administración Pública que, entre otras notas características se ve decisivamente influenciada por la consolidación, la ampliación, la diversificación y hasta la democratización de los servicios públicos en favor de los ciudadanos, mediante la asunción de objetivos que se han identificado bajo la denominación de procura existencial por parte del Estado, lo que conduce a la inserción de categorías tales como la eficiencia en la consideración del actuar administrativo público ${ }^{5}$.

Tal etapa se ve rápidamente complementada y hasta podría decirse sustituida por la que deviene de los avances tecnológicos más recientes, conducentes a la Sociedad Tecnológica y que trae consigo un correlativo proceso de ajuste o adaptación de la Administración Pública, consistente fundamentalmente en la incorporación decisiva de técnicas y mecanismos novedosos para el conocimiento de los asuntos, el almacenamiento, manejo y transferencia de la información, la mayor capacidad de respuesta, y en fin la elevación de las condiciones para dar satisfacción a los ciudadanos, los cuales también, en paralelo, elevan los rangos de sus demandas y exigen mayor calidad en las respuestas requeridas para la satisfacción de sus necesidades ${ }^{6}$.

\footnotetext{
${ }^{5}$ Al respecto puede verse Ernst Forsthoff, Sociedad Industrial y Administración Pública. Escuela Nacional de Administración Pública. Madrid.

${ }^{6}$ Véase, Armando Rodríguez García, «Descentralización del Poder y nuevas Tecnologías», en XXIV Congreso Iberoamericano de Municipios. Guadalajara. OICI/FEMP, Madrid 1998, pp. 135 y stes.
} 
Todo lo antes dicho permite comprender y reafirmar el carácter seminal que la noción de servicio público ha tenido y tiene en el ámbito del Derecho Administrativo.

No obstante, conviene tener presente que, tal como sucede con otros muchos conceptos juridificados, no se trata de una noción originada en el mundo jurídico, sino en la realidad de la dinámica social, como mecanismo de respuesta a la satisfacción de las necesidades colectivas de los individuos, a partir del momento en que se arriba a un cierto nivel de complejidad en las comunidades, y en atención a la presencia de valores relativos a la calidad de vida de sus pobladores.

En tal sentido, el papel que corresponde jugar a la jurisdicción contenciosoadministrativa va más allá del mero control de la legalidad, la protección singularizada de las situaciones jurídicas involucradas en un determinado conflicto con la Administración, o la determinación de responsabilidad pública derivada de circunstancias vinculadas con la actividad de servicio público. El órgano jurisdiccional puede tener un rol decisivo en la conceptualización, adaptación y modernización de la noción de servicio público, y ello tendrá estrecha relación con la concepción de sociedad que se tenga, con los valores que se intente instalar y preservar, y sobre todo, con el conocimiento que sobre las características y la realidad del fenómeno pueda manejar, para superar así, la expresión básica de control de legalidad, dando cabida a un papel de agente dinamizador de la disciplina jurídica, en función del desarrollo y la evolución de la sociedad.

\section{SERVICIO PÚBLICO, CIVILIZACIÓN, URBANIZACIÓN, TECNOLOGÍA Y CALIDAD DE VIDA}

Desde sus orígenes, y mucho más claramente en la actualidad, los servicios públicos son, en su esencia, una clara expresión de tecnología, y precisamente, a partir de esta nota, aparece un vínculo significativamente importante entre las nociones de servicio público, gestión pública y derecho administrativo, comprendiendo en ésta última noción toda sus posibles expresiones, y en particular, a los fines que nos interesan más directamente en esta oportunidad, la jurisdicción contencioso administrativa, tanto por lo que respecta a la actividad de control de la legalidad de los actos, como por la protección de los derechos e intereses colectivos e individuales asociados con las situaciones y vinculaciones de los diferentes actores (instituciones públicas, empresas, usuarios, etc.).

De otra parte, la evolución misma de los elementos tecnológicos y su difusión, en atención a los avances en las comunicaciones, ha permitido una sólida transferencia de información, que impregna a los miembros de las 
POTENCIALIDAD DEL CONTENCIOSO ADMINISTRATIVO EN ... LOS SERVICIOS PÚBLICOS

comunidades del conocimiento más actualizado y preciso sobre variedad y calidad de servicios, ampliando de manera prácticamente ilimitada el volumen de demandantes o usuarios reales y potenciales de servicios existentes, así como de nuevos servicios; demanda ésta que crece en forma estable, sostenida y segura.

De su parte, el espectro más amplio y significativo de prestación y utilización de los servicios públicos, alcanza su máxima expresión de tangibilidad en los espacios urbanos, esto es, dentro del sistema de vida urbano adoptado por la amplia mayoría de la población.

Es el ambiente urbano el que permite ofrecer de manera más eficiente, el mayor volumen de satisfacción a las necesidades colectivas, con los menores costos, en razón de las economías de escala.

Esta realidad incorpora otro dato de interés para la comprensión y adecuado tratamiento del tema de los servicios públicos. Se trata de la cuestión relativa a la categoría de Administración que se asocia al asunto, y que pone en lugar prioritario a la Administración Local, pero regularmente en forma combinada (no excluyente) con otras entidades territoriales, con las consecuencias que ello comporta en materia de control jurisdiccional, en función de la distribución de competencias por razón del sujeto.

Además, en atención a esta misma circunstancia, aparecen un conjunto de situaciones y relaciones interinstitucionales que provocan aspectos de interés para el tratamiento jurisdiccional, con lo cual aumenta la riqueza de oportunidades que el tema de los servicios públicos genera.

En cuanto al escenario físico y funcional, no hay duda respecto a que la ciudad ha sido, es, y evidentemente continuará siendo por mucho tiempo, el más formidable complejo tecnológico y la mayor hazaña cultural de la humanidad, que por sus características comporta el mayor grado de dinamismo social, económico y político, siendo una de sus expresiones básicas, la de presentar los niveles de confort y calidad de vida más avanzados que conoce la población, y con ello, conformar el espacio de concentración más importante de la multiplicidad de servicios públicos que conoce la sociedad en el momento actual.

Pero a la evolución brevemente reseñada hace falta agregar un dato más reciente de indudable relevancia, constituido por la llamada Revolución Tecnológica que opera con mayor difusión y velocidad que la ya citada Revolución Industrial en las postrimerías del recién finalizado siglo Xx, y que impacta fundamentalmente las áreas de las comunicaciones y la información, básicamente a través de los avances en materia informática y electrónica que se ponen en muy breve plazo a disposición de incontables contingentes de población.

La transformación tecnológica, así como el incremento en la calidad y velocidad de las comunicaciones y la información, hacen que se irradie el 
conocimiento de las nuevas ofertas de servicios, ampliando la potencialidad de su demanda a nuevos contingentes de usuarios en escalas antes desconocidas.

Pero los servicios públicos no sólo se amplían cuantitativamente, es decir, en cuanto al numero de usuarios, sino que también se produce una variación constante y sensible en su calidad y en cuanto a las modalidades de prestación.

El abastecimiento de agua, por ejemplo, que comienza por ser un suministro en puntos de abastecimiento colectivo, se transforma en un servicio individualizado para cada unidad inmobiliaria, pero además se potabiliza el líquido, para permitir el consumo humano, sin riesgos de salud. En materia de comunicaciones, en un plazo relativamente corto, se ha pasado de la prestación del servicio telefónico mediante una central o estación de comunicación a cargo de un operador, para servir a toda una población, a la prestación directa del servicio en la vivienda, añadiendo seguidamente las unidades auxiliares, los servicios de discado directo y larga distancia, la telefonía satelital, y hoy en día es extraño voltear a cualquier parte en un espacio público y no encontrar una o varias personas comunicándose mediante telefonía móvil.

Todos estos cambios y transformaciones se producen fundamentalmente en el medio urbano y como efecto de las actividades desplegadas en él, incluyendo los nuevos modos de gestión y operación, que desde luego, se transfieren a fórmulas jurídicas.

La Administración contemporánea debe superar ampliamente los conceptos que la han venido moldeando desde su formulación inicial, para obtener el perfil atlético y ágil con el cual pueda dar satisfacción eficiente y de calidad a la población. El Profesor Valentín MERINO afirma sobre esta asunto que: «... En España, como en otros países europeos con notable desarrollo del estado de bienestar, la valoración de los servicios públicos ha mejorado notablemente en los últimos años. Pero aún se percibe a la Administración Pública como un abultado aparato burocrático, lento e ineficiente, poco receptivo a las demandas ciudadanas. En muchos países iberoamericanos, a esto se añade una debilidad estructural de la Administración, así como notables problemas de falta de equidad y objetividad, en la percepción de grandes sectores de la población. En general, la Administración Pública aparece cuestionada principalmente por su ineficiencia, pues no justifica en términos de rendimientos, la gran cantidad de recursos que absorbe de la sociedad ${ }^{7}$.

Esa percepción obliga a formular respuestas desde diferentes frentes, dentro de los cuales, desde luego ocupa lugar preponderante, la propia organización

\footnotetext{
${ }^{7}$ Valentín Merino Estrada, «Calidad Total en la Gestión de la Administración Municipal». Derecho Administrativo y Municipio. II Jornadas de Derecho Administrativo. Universidad Católica del Táchira. San Cristóbal , 2005, p. 139.
} 
POTENCIALIDAD DEL CONTENCIOSO ADMINISTRATIVO EN ... LOS SERVICIOS PÚBLICOS

administrativa, en su posición de responsabilidad inmediata y capacidad de reacción eficiente de primer orden frente a los nuevos escenarios en los cuales debe desenvolverse. «...Los principios y prácticas de la Gestión de Calidad Total son plenamente aplicables en la Administración Pública. Es más, aplicarlos constituye una necesidad ineludible...La Administración precisa una estrategia de calidad orientada al ciudadano-cliente, para modernizarse y fortalecer su legitimidad» ${ }^{8}$.

Pero la Administración no es el único complejo organizativo que puede actuar en la necesaria modernización de los criterios y consecuente adaptación de estructuras y métodos para lograr la generación de «productos» acordes con el momento. También los órganos jurisdiccionales tienen parlamentos importantes de pronunciarse en voz alta dentro del guión de la dinámica social contemporánea y sus perspectivas de evolución inmediata.

La Constitución venezolana de 1999, al consagrar la jurisdicción contencioso administrativa (artículo 259), mantiene la magistral fórmula que instaló el artículo 206 del texto precedente de 1961, añadiendo como parte de la amplitud sustantiva de esta expresión de la función jurisdiccional, la siguiente referencia: «...conocer de reclamos por la prestación de servicios públicos...».

En nuestro criterio, esta frase resulta irrelevante, en cuanto al alcance del contencioso administrativo en materia de servicios públicos, pues salvo el hecho de que hace expresa una capacidad de actuación que, sin lugar a dudas, ya tenía el juez contencioso administrativo, no ofrece mayor novedad, como no sea realzar la singularización del servicio público como materia objeto de control. No obstante, es oportuno observar al respecto que la mención resulta absolutamente mezquina y limitada, cuando se circunscribe a reclamos «por la prestación» de los servicios, lo cual seguramente tendrá una interpretación de carácter progresivo, que deberá incluir como alcance, entre otros aspectos, lo relativo a la instalación y modernización de los servicios.

Sobre el particular recuerda DROMI, que «El bien común, ratio essendi del Estado, lleva implícita de un modo prioritario la integridad prestacional que importa calidad en la gestión de los servicios públicos (...) En nuestros días, la calidad de vida importa que los usuarios tengan asegurado el derecho a un nivel de prestación adecuado, tendiente al mejoramiento de las condiciones de su existencia, con servicios públicos eficientes, cuya prestación integral y acceso universal estén garantizados por el Estado, que debe, a través de las autoridades, proveer el control de calidad, la eficiencia y la regularidad...» ${ }^{9}$.

\footnotetext{
${ }^{8}$ Ibídem, p. 145

${ }^{9}$ Roberto Dromi, El Derecho Público en la Hipermodernidad. Editorial Hispania Libros. Madrid, 2005, p. 304.
} 
La sensibilidad y formación del juez contencioso administrativo permitirá entender como punto de partida, la necesaria adaptación de los moldes conceptuales a las realidades presentes en la actualidad, que en el campo de los servicios públicos, está decisivamente inmerso en la conceptualización de la vida urbana contemporánea. En palabras de Alfonso VegARA y José Luis DE LAS RIVAS: «En los albores del siglo XXI están apareciendo situaciones nuevas en todas las facetas de nuestra vida: El desarrollo de las nuevas tecnologías de la información y de las comunicaciones multiplica exponencialmente la capacidad de interacción global a todos los niveles; la apertura de los mercados internacionales introduce nuevas pautas de comportamiento en las empresas que operan globalmente; la actual estructuración política mundial hace difícil abordar de forma justa y coherente la redistribución de los recursos que genera la economía internacional y eso acentúa las desigualdades (...) Todos estos cambios afectan también a las ciudades y a la forma de gestionarlas...» ${ }^{10}$.

Todo ello invita, entonces, a reconocer la prevalencia que sobre las concepciones estrictamente jurídicas, tienen los componentes esenciales de los fenómenos a los cuales se aplican tales categorías jurídicas y familiarizarse con sus características, para poder colocarse en condiciones de aplicarla mejor y mas eficientemente. En materia de servicios públicos, es imprescindible reconocer las características del fenómeno de la urbanización y sus manifestaciones, con lo cual se podrá potenciar eficientemente la presencia de las decisiones jurisdiccionales para continuar construyendo una disciplina vigorosa y sobre todo sintonizada con la realidad para el mejor servicio al ciudadano en el escenario que se ha construido, y que constituye la mayor hazaña de la civilización: la ciudad.

Tal como señala el Profesor Fernando MANERo: «...Una de las implicaciones espaciales más significativas del proceso de globalización contemporánea consiste en la acelerada readaptación de las realidades urbanas a la lógica de la competitividad inducida por la liberalización de las relaciones comerciales y por la necesidad de afianzamiento de las ventajas comparativas de las ciudades. No cabe duda que en un contexto abierto a la movilidad plena de los factores, y singularmente del capital, cobran su máxima expresión las fuerzas favorecedoras de una consideración selectiva de los espacios ...» ${ }^{11}$.

\footnotetext{
${ }^{10}$ Alfonso Vegara y Juan Luis De las Rivas, Territorios Inteligentes. Ed. Fundación Metrópoli. Madrid, 2004, p. 291.

${ }^{11}$ Fernando Manero Miguel, «La gestión de la calidad de los espacios urbanos: un factor de competitividad territorial e integración espacial». En XXV Congreso Iberoamericano de Municipios. OICIFEMP. Madrid, 2003, p. 361.
} 